\title{
Multiple Imaging of Pulsars by the ISM
}

\author{
Yashwant Gupta \\ National Centre for Radio Astrophysics (TIFR), Pune, India
}

\begin{abstract}
Refraction of pulsar radiation by electron density irregularities in the interstellar medium can sometimes produce multiple imaging of pulsars. Such events can be used as tools to resolve the emission regions in pulsar magnetospheres. Results from recent observations of a double imaging event for PSR B1133+16 are described. Significant fringe phase variations are seen across the pulse. The minimum value for the transverse separation of the emitting regions at the two edges of the pulse is inferred to be $\approx 5 \times 10^{5} \mathrm{~m}$. These results are compared with earlier values reported in literature and the implications are discussed.
\end{abstract}

\section{Introduction}

Random electron density fluctuations in the interstellar medium (ISM) produce interstellar scintillation (ISS) of the radio signals received from pulsars. The small scale irregularities $\left(10^{5}\right.$ to $\left.10^{6} \mathrm{~m}\right)$ in the ISM produce a scatter broadened image of the pulsar, characterised by the diffractive scattering angle, $\theta_{d}$. The large scale irregularities $\left(\approx 10^{11} \mathrm{~m}\right)$ steer the diffractive scattering cone by an overall mean direction of arrival, $\theta_{r}$, expected to vary on refractive scintillation time scales. In dynamic spectra observations of pulsars, diffractive scintillation shows up as the random modulation of intensity (scintles) across the time-frequency plane, whereas the fixed direction of drift of the scintles at a single epoch is due to the refractive steering.

Sometimes, strong refraction effects in the ISM can produce two or more scatter broadened images of the pulsar with well separated directions of arrival, $\theta_{r 1}$ and $\theta_{r 2}$. Such multiple imaging events can produce additional features in pulsar dynamic spectra data, such as multiple drift slopes and periodic intensity modulations or fringes, over and above the random modulations due to diffractive scintillation.

Study of multiple imaging events allows a better understanding of the distribution of electron density irregularities in the ISM, particularly at large scales. See Rickett (this volume) for a detailed discussion of these issues. See also Rickett et al. (this volume) for a good example of the application of these techniques to a multiple imaging event for PSR B0834+06.

Multiple imaging events provide an exciting technique for studying pulsar emission geometry, as they allow the possibility of resolving the pulsar magnetosphere. This happens because the phase of the fringes is sensitive to the transverse location of the source of radiation. This method was first used by Wolszczan \& Cordes (1987) to resolve the emission region for PSR B1237+25 and 




Figure 1. Dynamic spectra data for PSR B1133+16 on April 30, 1994

later on by Wolszczan et al.(1988) for PSR B1133+16 and by Kuz'min (1992) for PSR B1919+21. From measurements of the phase change of the fringes from the leading to the trailing edge of the pulse, these authors report the transverse separation of the emission regions in the magnetosphere to be $\approx 5 \times 10^{6} \mathrm{~m}$ to $3 \times 10^{7} \mathrm{~m}$. New results for PSR B1133+16 using data recorded the Ooty Radio Telescope (ORT) are presented here.

\section{New Observations of Multiple Imaging of PSR B1133+16}

As part of a regular program for monitoring slow variations of diffractive scintillation properties of pulsars due to refractive scintillation, PSR B1133+16 was observed at 27 epochs from March to May 1994, using the ORT. The observations were carried out at a frequency of $327 \mathrm{MHz}$ with a $9 \mathrm{MHz}$ bandwidth spanned by 64 spectral channels. The dynamic spectra data were generated by on-line averaging of 10 successive pulses, leading to an effective time constant $\approx$ 12 secs. The data were gated around the on-pulse phase of the pulsar signal and a time resolution of $6 \mathrm{msec}$ was maintained, thereby providing phase resolved dynamic spectra with a resolution of 1.8 degrees of longitude.

Dynamic spectra data from one day - 30 April 1994 - showed clear periodic modulations of the intensity (figure 1), indicating that an epoch of multiple imaging had been detected. Secondary spectral analysis of this dynamic spectra data showed the periodicity to be $\nu_{t}=5.9 \times 10^{-3}$ cycles $/ \mathrm{sec}$ and $\nu_{f}=1.6 \mathrm{cycles} / \mathrm{MHz}$ in the time and frequency domains, respectively. Data taken two days earlier and two days later did not show any signs of such fringes. 

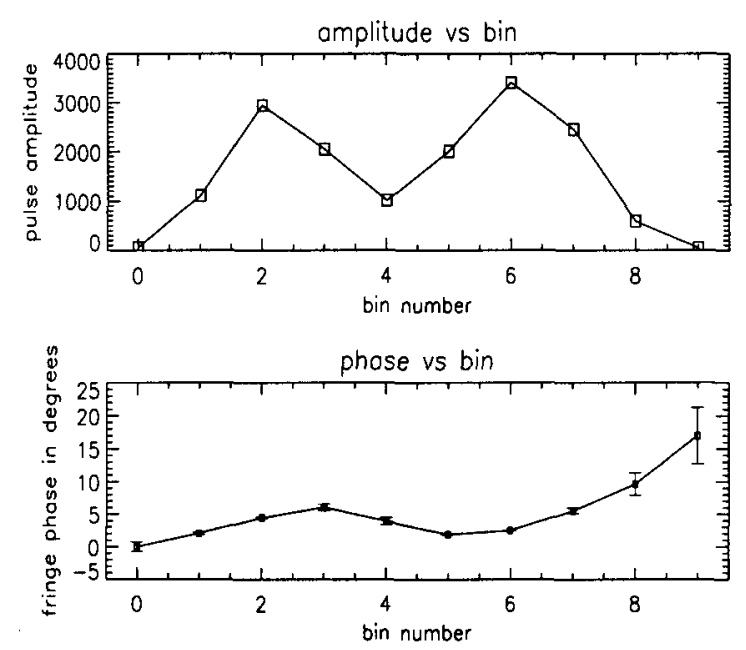

Figure 2. Variation of amplitude and fringe phase across the pulse

There were 10 time samples across the pulse width for this observation. Analysis showed the fringes to be present in each of these phase resolved dynamic spectra, with the amplitude of the periodicity following the mean pulse shape. The total variation of the phase of the fringes across the pulse $(\Delta \phi)$ was found to be $\approx 15^{\circ}$ (figure 2 ), showing that the emission region was resolved during these observations. The phase variation was, however, not monotonic, showing two local extrema within the pulse.

\section{Modelling and Interpretation of the Observations}

Interpretation of the observed variation of the fringe phase across the pulse as separation of the corresponding emission regions depends critically on the understanding of the underlying geometry. This involves the following vector quantities (all projected onto the plane of the sky) : $\theta_{r 1}$ and $\theta_{r 2}$ - the two refractive angles; $\mu_{p m}$ - the pulsar proper motion vector; and $\Delta s$ - the vector representing the transverse separation between the leading and trailing edges of the emitting regions when they are radiating towards the observer (figure 3 ). In general, the angles between all these vectors are unknown.

The equations that apply to this situation are as follows:

$$
\begin{aligned}
\nu_{t}=\frac{f_{0} L_{p} \mu_{p m} \theta_{12} \cos (\alpha)}{c} & =\frac{f_{0} L_{p} \mu_{p m}\left(\theta_{r 1} \cos \left(\alpha_{1}\right)-\theta_{r 2} \cos \left(\alpha_{2}\right)\right)}{c}, \\
\nu_{f} & =\frac{3 L_{p}\left(\theta_{r 1}^{2}-\theta_{r 2}^{2}\right)}{4 c} \\
\Delta \phi & =\frac{2 \pi \nu_{t} \Delta s \cos (\gamma-\alpha)}{L_{p} \mu_{p m} \cos (\alpha)}
\end{aligned}
$$




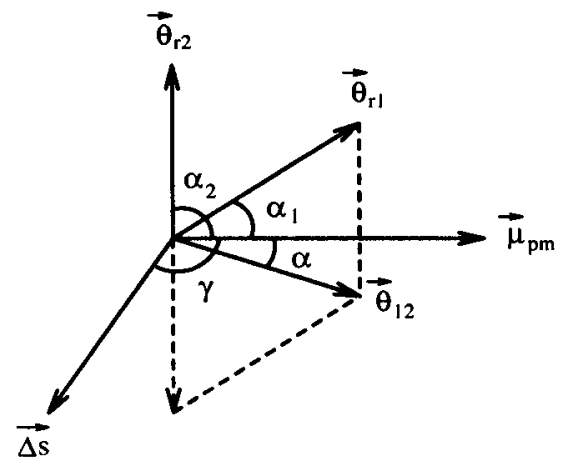

Figure 3. Illustration of the geometry for multiple imaging

Here $f_{0}$ is the observing frequency, $L_{p}$ is the pulsar distance and $c$ is the speed of light. The various angles are as shown in figure 3. This set of equations is similar to that given by Cordes \& Wolszczan (1988). Assuming $L_{p}, f_{0}$ and $\mu_{p m}$ to be known quantities, there are 6 independent unknown quantities $-\theta_{r 1}, \theta_{r 2}$, $\Delta s, \alpha_{1}, \alpha_{2}$ and $\gamma-$ and only 3 equations. Thus, extra input is needed to invert the problem and obtain a solution for $\Delta s$. Some information can be obtained from the slope of the drift bands produced by the two images contributing to the dynamic spectra, by using the relation between the drift slopes, the refractive angles and the proper motion. The two relevant equations are

$$
\left.\frac{d t}{d f}\right|_{1}=-\frac{\theta_{r 1} \cos \left(\alpha_{1}\right)}{f_{0} \mu_{p m}} ;\left.\frac{d t}{d f}\right|_{2}=-\frac{\theta_{r 2} \cos \left(\alpha_{2}\right)}{f_{0} \mu_{p m}} .
$$

The measured values for the slopes are $1.8 \times 10^{-4} \mathrm{sec} / \mathrm{Hz}$ and $-5.0 \times 10^{-4} \mathrm{sec} / \mathrm{Hz}$ for the data of figure 1 . Better constraints can now be placed on the values of the unknown quantities. For example, for a given choice of one of the unknowns say, $\theta_{r 1}$, unique values can be calculated for $\alpha_{1}, \theta_{r 2}, \alpha_{2}$ (and hence $\alpha$ ). Then; assuming the extreme case of $\gamma=\alpha$ allows the calculation of a lower limit to $\Delta s$ for the given choice of $\theta_{r 1}$ as

$$
\Delta s_{\min }=\frac{\Delta \phi L_{p} \mu_{p m} \cos (\alpha)}{2 \pi \nu_{t}} .
$$

This procedure has been followed for a plausible range of values for $\theta_{r \cdot 1}$ given by $\left|\theta_{r 1}\right|<10 \theta_{d}$. The smallest values for $\Delta s_{\min }$ found are $\approx 5 \times 10^{5} \mathrm{~m}$ and are achieved for $\theta_{r 1}, \theta_{r 2}$ values $\approx 5-10$ mas. Using a dipole geometry model for the pulsar emission, this value translates to an emission altitude of $\approx 1 \times 10^{7} \mathrm{~m}$, which is $20 \%$ of the light cylinder radius for this pulsar.

\section{Conclusions and Discussion}

The value for $\Delta s$ and emission altitude estimated here provide the smallest lower limits of all currently available estimates using the technique of multiple imaging. Furthermore, it is the only value that is also consistent with the upper 
limit of $\Delta s<1 \times 10^{6} \mathrm{~m}$ obtained for this pulsar from diffractive scintillation techniques (Cordes et al., 1983). The estimated emission altitude is much larger than that obtained using other techniques such as multifrequency timing and polarisation and pulse morphology studies (e.g. Phillips, 1992). An interesting possibility that can resolve this disagreement has been presented in a poster in this meeting by Gallant.

In conclusion, further data of multiple imaging events (preferably multiple epochs for a pulsar) with careful measurements of drift slopes in the dynamic spectra are required to obtain better constraints on the pulsar emission geometry. More work is also needed to understand the detailed shape of the curve of fringe phase versus pulse longitude.

Acknowledgments. The author wishes to thank Ramesh Bhat for the data acquisition and help with the analysis and A.P.Rao for valuable discussions.

\section{References}

Cordes, J.M., Weisberg, J. \& Boriakoff, V. 1983, ApJ, 268, 370

Cordes, J.M. \& Wolszczan, A. 1988, in "AIP Conference Proceedings \# 174" ed. J.M. Cordes, B.J. Rickett \& D.C. Backer (New York : AIP), 212

Kuz'min, O.A. 1992, in "The Proceedings of IAU Colloquium \# 128" ed. T.H. Hankins, J.M. Rankin \& J.A. Gil (Zielona Gora, Poland: Pedagogical Univ. Press), 288

Wolszczan, A., Bartlett, J.E. \& Cordes, J.M. 1988, in "AIP Conference Proceedings \# 174" ed. J.M. Cordes, B.J. Rickett \& D.C. Backer (New York : AIP), 145

Wolszczan, A. \& Cordes, J.M. 1987, ApJ, 320, L35 